\title{
Plasma neutrophil gelatinase-associated lipocalin predicts acute pyelonephritis in children with urinary tract infections
}

\author{
Ji Hyun Sim', Hyung Eun Yim', Byung Min Choi', Jee Hyun Lee' and Kee Hwan Yoo'
}

BACKGROUND: The identification of acute pyelonephritis (APN) is still a challenge.

METHODS: Patients admitted for their first urinary tract infection (UTI) were enrolled. Plasma neutrophil gelatinaseassociated lipocalin (NGAL) levels were measured at admittance and after treatment. Laboratory, clinical, and imaging results were compared between children with and without APN.

RESULTS: A total of 123 patients were enrolled (53 APN and 70 lower UTI). After adjusting for age and gender, plasma NGAL levels were higher in the APN group than in the lower UTI group (233 (129-496) ng/ml vs. $71(50.8-110) \mathrm{ng} / \mathrm{ml}, P<$ $0.001)$. NGAL levels were correlated with the serum levels of leukocytes, C-reactive protein, and creatinine, as well as fever duration $(P<0.05)$. Multivariable analysis revealed that logtransformed plasma NGAL was an independent predictor of APN $(P<0.05)$. Receiver operating curve analysis showed a good diagnostic profile of NGAL for identifying APN (area under the curve 0.864 ) with a best cut-off value of $102.5 \mathrm{ng} / \mathrm{ml}$. The NGAL levels in both two groups decreased after treatment compared to levels before treatment $(P<0.001)$.

CONCLUSION: Plasma NGAL can be a sensitive predictor for identifying APN and monitoring the treatment response of pediatric UTI.

U rinary tract infections (UTIs) are among the most common bacterial infections in children (1). Early detection and proper management of UTIs is important since UTIs involving the kidney may cause permanent renal scarring and be a risk factor for future development of renal insufficiency (2). Establishing the diagnosis of acute pyelonephritis (APN) requires imaging with $99 \mathrm{~m}$ Tc-dimercaptosuccinic acid (DMSA) scintigraphy to view renal parenchymal involvement (3). Although a DMSA scan is considered to be very sensitive in the assessment of renal damage, it is expensive, not readily available in all centers, and exposes the patients to radiation (4). Therefore, a more practical and accessible tool to determine the presence of renal parenchymal involvement could be helpful for the timely management of UTIs.

Neutrophil gelatinase-associated lipocalin (NGAL), as a member of the lipocalin superfamily, is produced from the nephron in response to tubular epithelial damage (5). It has been identified as one of the earliest and potentially one of the most indicative biomarkers of acute kidney injury (AKI) from a diverse array of conditions and can differentiate between prerenal and intrinsic causes $(6,7)$. Urinary NGAL (uNGAL) levels increase in patients with different causes of nephropathies $(8,9)$ and might be a valuable tool in monitoring the treatment response of various renal diseases $(10,11)$.

As an iron-carrier protein derived from human neutrophils, NGAL also plays an important role in the innate immune response to bacterial infection (12). Experimentally, NGAL was capable of blocking Escherichia coli growth under ironpoor conditions, but NGAL with siderophore iron was not (13). NGAL-knockout mice demonstrated substantially increased mortality after intraperitoneal infection with E. coli (12). In humans, plasma NGAL has been reported to be a useful predictor for distinguishing acute bacterial infections from viral infections in populations from newborns to adults (14-16). Recent studies have also shown a possible association between increased NGAL levels and UTIs. Ichino et al. (17) demonstrated experimentally that uNGAL levels in rats increased in both the early and extensive fibrosis stages of pyelonephritis, suggesting that UNGAL might also be useful as a marker of APN and renal scarring in humans. Indeed, we, and other investigators, have demonstrated that UNGAL can be a useful predictor for detecting UTIs in children $(1,18)$. In patients with febrile UTIs, increased UNGAL levels were related to UTI severity, and levels decreased following antibiotic treatment. The study results suggested that uNGAL could be more accurate than other biomarkers, such as urinary kidney injury molecule- 1 and serum cystatin C, for diagnosing and assessing febrile UTIs (18). In our preliminary study, increased plasma NGAL levels were also highly associated with febrile UTIs in infants aged 1-12 mo (19). However, few studies have addressed whether NGAL has promise for detecting APN in patients with UTIs, and further research is required to define NGAL's role in routine clinical practice.

The objectives of this study were to assess whether plasma NGAL could be a promising diagnostic biomarker of renal parenchymal involvement in children with symptomatic UTI and to determine the correlation with other parameters

'Department of Pediatrics, College of Medicine, Korea University, Seoul, South Korea. Correspondence: Hyung Eun Yim (he-yim@hanmail.net)

Received 25 July 2014; accepted 22 December 2014; advance online publication 15 April 2015. doi:10.1038/pr.2015.59 
related to APN and late renal scarring. In comparison to a previous preliminary study (19), in this study, we increased the sample size and the range in age and symptoms of enrolled patients.

\section{RESULTS}

\section{Patient Demographics}

Among 195 patients with UTIs, 72 were excluded and 123 were enrolled with their first episode of symptomatic UTI (Figure 1). Fifty-three patients were included in the APN group and 70 patients in the lower UTI group. There was no difference in the median age between the two groups. There was a higher distribution of males in the lower UTI group (APN vs. lower UTI, 56.6 vs. 74.3\%, $P<0.05$ ). After adjusting for age and gender, serum levels of leukocyte counts, $\mathrm{C}$-reactive protein $(\mathrm{CRP})$, and creatinine $(\mathrm{Cr})$ were significantly higher in the APN group compared to those in the lower UTI group $(P$ $<0.05)$. No patients had specific signs or symptoms of AKI. Fever duration before antibiotic treatment was longer in the APN group than the lower UTI group $(P<0.05)$. There was no difference in the presence of hydronephrosis and vesicoureteral reflux (VUR) between the two groups. Twenty-five of 53 patients with APN underwent a follow-up DMSA scan 5-6 mo after the study, and 9 patients had persistent abnormalities, suggesting renal scarring (Table 1).

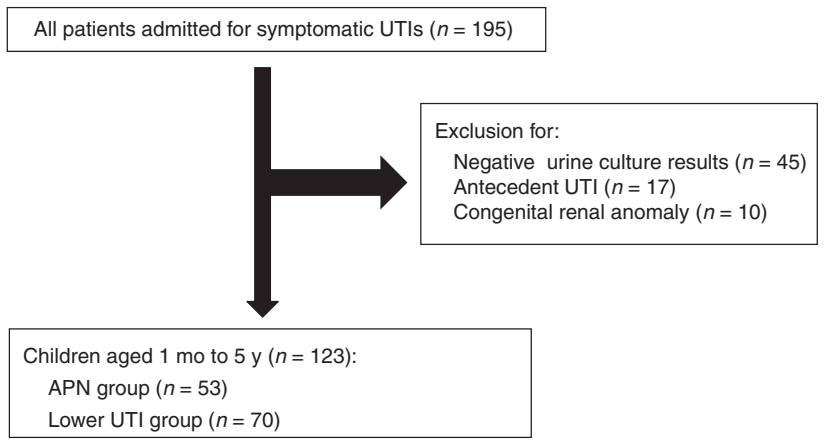

Figure 1. Study flow diagram.

\section{NGAL Quantification}

After adjusting for age and gender, plasma NGAL levels were significantly higher in patients with APN than in those with lower UTI (233 (interquartile range $(\mathrm{IQR})=129-496) \mathrm{ng} /$ $\mathrm{ml}$ vs. $71(\mathrm{IQR}=50.8-110) \mathrm{ng} / \mathrm{ml}, P<0.001)$. Between 4 and $5 \mathrm{~d}$ after antibiotic treatment, the median plasma NGAL levels significantly decreased in both groups (APN 233 (IQR $=129-496) \mathrm{ng} / \mathrm{ml}$ vs. $88.5(\mathrm{IQR}=62.5-119) \mathrm{ng} / \mathrm{ml}$; lower UTI $71(\mathrm{IQR}=50.8-110) \mathrm{ng} / \mathrm{ml}$ vs. $42(\mathrm{IQR}=32-65) \mathrm{ng} / \mathrm{ml}$, $P<0.001)$. The median plasma NGAL levels after antibiotic treatment were also higher in patients with APN than in those with lower UTIs $(P<0.001)$ (Figure 2a). The comparisons of log-transformed plasma NGAL levels between the two groups showed similar findings with those of plasma NGAL levels (Figure 2b). Log plasma NGAL levels were strongly correlated with log-transformed serum CRP concentrations, the serum levels of leukocytes and $\mathrm{Cr}$, and fever duration before admission, respectively (all $P<0.05$ ) (Figure $3 \mathrm{a}-\mathrm{d}$ ). There were no differences in plasma NGAL levels between patients with and without scars and between those with and without VUR (data not shown).

\section{Univariate and Multivariate Logistic Regression Analyses}

Univariate analysis identified several clinical variables associated with the presence of APN, as shown in Table 2. Gender, fever duration before antibiotic treatment, serum white blood cells (WBCs), serum Cr, log-transformed serum CRP, and log-transformed plasma NGAL were significant; however, in a multivariate stepwise logistic regression analysis, only logtransformed plasma NGAL $(P=0.007)$ and serum leukocyte counts $(P=0.04)$ were retained as significant predictors of APN (Table 2).

\section{Receiver Operating Curve (ROC) Analyses}

ROC analyses were performed to define the diagnostic profile of plasma NGAL in patients with UTIs. According to ROC analysis, the optimal cut-off level for predicting APN with plasma NGAL was $102.5 \mathrm{ng} / \mathrm{ml}$. Using this cutoff value, the plasma NGAL had a sensitivity of $89.1 \%$ and

Table 1. Study population characteristics

\begin{tabular}{lccc}
\hline Variables & APN & Lower UTI & $P$ value \\
\hline Subjects, $n$ (\%) & $53(43.1)$ & $70(56.9)$ & - \\
Age (months) & $4(3-5.3)$ & $4(3-6)$ & 0.709 \\
Male gender, $n$ (\%) & $30(56.6)$ & $52(74.3)$ & 0.039 \\
Fever duration (days) & $2(1-4)$ & $1(1-2)$ & 0.005 \\
Serum WBCs $/\left(\mathrm{mm}^{3}\right)$ & $17,770(12,460-22,178)$ & $13,350(11,100-16,100)$ & $<0.001$ \\
Serum CRP $(\mathrm{mg} / \mathrm{dl})$ & $6.78(4.56-13.5)$ & $2.57(0.9-4.45)$ & $0.31 \pm 0.10$ \\
Serum Cr $(\mathrm{mg} / \mathrm{dl})$ & $0.37 \pm 0.13$ & $27 / 70(38.6)$ & 0.002 \\
Hydronephrosis, $n(\%)$ & $23 / 53(43.4)$ & $7 / 51(13.7)$ & - \\
VUR, $n(\%)$ & $15 / 52(28.8)$ & - & 0.001 \\
Renal scarring, $n(\%)$ & $9 / 25(36.0)$ & - \\
\hline
\end{tabular}

$P$ values are adjusted for age and gender. Data are presented as numbers (proportions) or median (interquartile range) or mean \pm SD.

APN, acute pyelonephritis; CRP, C-reactive protein; UTI, urinary tract infection; VUR, vesicoureteral reflux; WBC, white blood cells. 

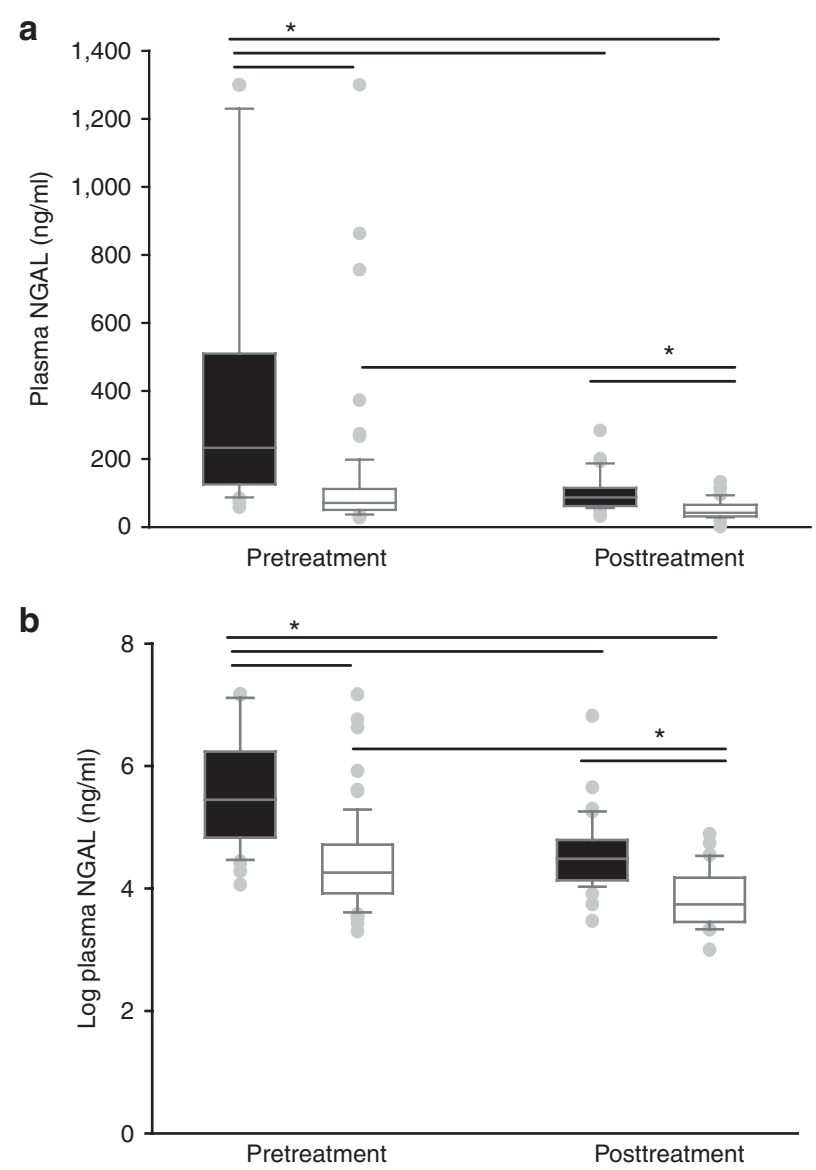

Figure 2. Comparison of median plasma neutrophil gelatinase-associated lipocalin (NGAL) levels. (a) Plasma NGAL levels before and after antibiotic treatment in each urinary tract infection (UTI) group. (b) Log-transformed plasma NGAL levels before and after antibiotic treatment in each UTI group. Boxes indicate the 25 th and 75 th percentiles, horizontal lines in each box indicate median values, whiskers indicate the 10th and 90th percentiles, and circles represent values outside this range (black box; acute pyelonephritis, white box; lower UTI, ${ }^{*} P<0.001$ ).

a specificity of $71.0 \%$ for a diagnosis of APN. The positive and negative predictive values of this cut-off value were 68.3 and $90.7 \%$, respectively. Using ROC analysis, the area under the curve (AUC) was 0.864 (95\% confidence interval (CI) $0.798-0.930, P<0.05)$, suggesting that plasma NGAL could be a good marker for diagnosing APN in children with UTIs (Figure 4a). To investigate the diagnostic accuracy between other biomarkers and APN, ROC analyses of serum CRP, WBCs, and Cr were performed. The AUC of ROC was 0.842 (95\% CI: 0.772-0.913) for CRP, 0.698 (95\% CI: 0.600-0.797) for WBCs, and 0.650 (95\% CI, 0.544-0.756) for Cr (all $P<0.05$ ) (Figure $4 \mathbf{b}-\mathbf{d}$ ). The cut-off values of CRP, leukocytes, and Cr for a diagnosis of APN were $3.86 \mathrm{mg} / \mathrm{dl}, 16,555 /$ $\mathrm{mm}^{3}$, and $0.33 \mathrm{mg} / \mathrm{dl}$ respectively. Among these parameters, plasma NGAL had the highest AUC value. The AUC difference between NGAL and CRP was not significant $(P=0.56)$, whereas the AUC differences between NGAL and WBC and between NGAL and Cr were statistically significant (all $P<0.05$ ) (Figure 4e).

\section{DISCUSSION}

The differentiation between APN and lower UTI is very important because renal parenchymal involvement can induce permanent renal scars that may lead to hypertension and chronic renal failure (2). However, the diagnosis of APN is still a challenge. Although DMSA renal scan is a valid tool for confirming the diagnosis of APN in children (3), the routine use of imaging for localization of UTI is not recommended (20). Furthermore, in adults, APN is differently defined with regard to imaging or clinical criteria and information on the relationship between imaging and clinical data is limited (21). The literature demonstrates that different diagnostic and treatment pathways are followed for adults compared with children even though Talner and et al. underlined the importance of standardizing the definition of APN about $20 \mathrm{y}$ ago $(22,23)$. The quest to improve accurate rapid diagnosis of APN is an area of intense research (24).

In the present study, plasma NGAL levels were significantly higher in children with APN than in those with lower UTIs and the levels in the APN and lower UTI groups decreased after antibiotic treatment compared to levels before treatment. Log plasma NGAL also significantly correlated with other parameters, including log serum CRP, serum levels of leukocyte counts and Cr, as well as fever duration before admission. Multivariate logistic regression analysis revealed that log plasma NGAL was an independent predictor of APN. These findings suggest that plasma NGAL could be a promising biomarker for detecting APN and monitoring the treatment response of pediatric UTI.

NGAL is a $25 \mathrm{kDa}$ protein originally purified from human neutrophils (25). It is considered a specific marker of neutrophil activity and a strong bacteriostatic agent, as it involves the antibacterial iron-depletion strategy of the innate immune system $(13,26)$. NGAL is expressed at low levels in normal organs and increases in injured epithelia, including in the lung, colon, and especially in the kidney $(11,27,28)$. The levels of NGAL are not raised in healthy full-term newborns at birth, but neutrophils from newborns, even premature infants, have been able to rapidly release NGAL upon bacterial or fungal stimulation in vivo (15). In children hospitalized with symptoms and signs of acute infections, NGAL is notably increased at admittance with bacterial infection than viral infection, suggesting that NGAL could be a valuable diagnostic marker for differentiating between acute bacterial and viral infections (16). It is, therefore, not surprising that we found a significant increase in urine and blood NGAL levels in children with UTIs $(18,19)$. Further, we observed that plasma NGAL levels could differentiate APN from a lower UTI and predict the treatment response of pediatric UTIs. In accordance with our findings, renal NGAL mRNA and protein levels have been shown to increase soon after bacterial injections in an experimental rat UTI model (17). Hatipoglu et al. (29) showed significantly higher levels of urine matrix metalloproteinase-9/NGAL/ $\mathrm{Cr}$ in children with UTIs compared to those in children with contaminated urine. The urine matrix metalloproteinase-9/ NGAL/Cr levels in children with UTIs were diminished after 
a

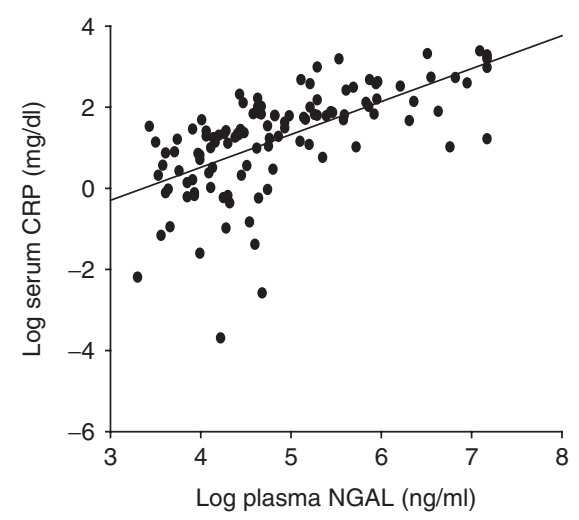

C

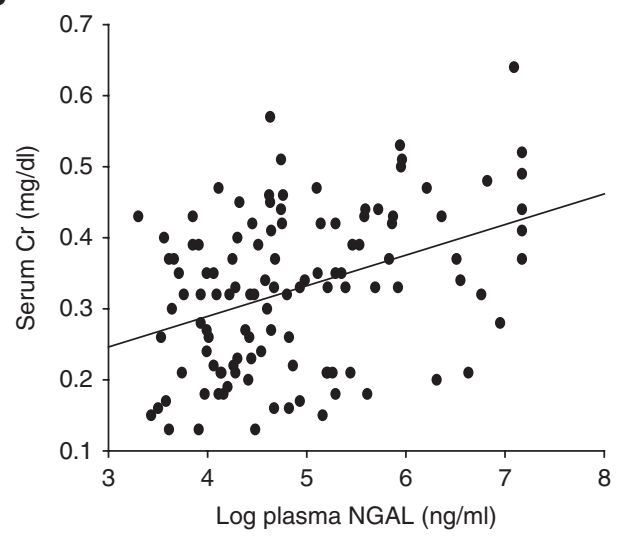

b

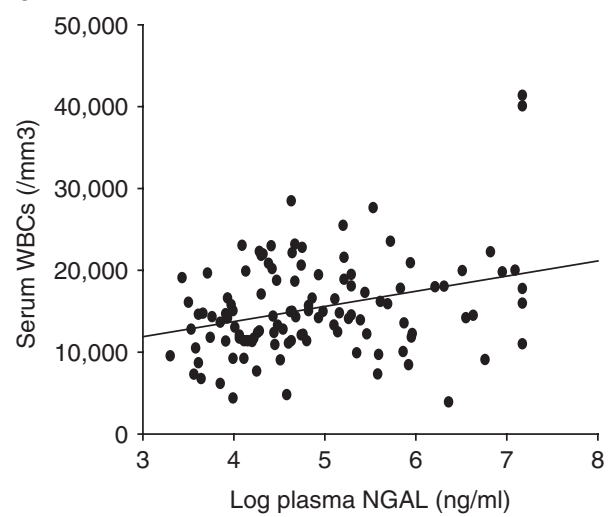

d

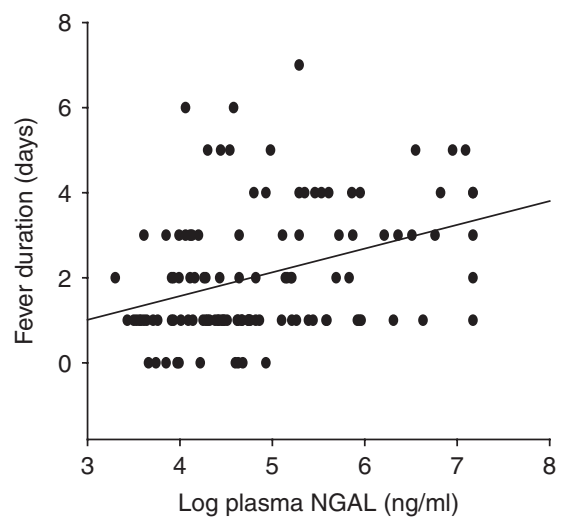

Figure 3. Correlations between log-transformed plasma neutrophil gelatinase-associated lipocalin and (a) Log-transformed serum C-reactive protein $(r=0.652, P<0.001)$, (b) Serum white blood cells $(r=0.32, P=0.001)$, (c) Serum $\mathrm{Cr}(r=0.378, P<0.001)$, (d) Fever duration before admission $(r=0.35$, $P<0.001)$.

Table 2. Univariate and multivariate logistic regression analyses of the variables associated with the presence of acute pyelonephritis

\begin{tabular}{|c|c|c|c|c|}
\hline \multirow[b]{2}{*}{ Variables } & \multicolumn{2}{|c|}{ Univariate analysis } & \multicolumn{2}{|c|}{ Multivariate analysis } \\
\hline & OR $(95 \% \mathrm{Cl})$ & $P$ value & OR $(95 \% \mathrm{Cl})$ & $P$ value \\
\hline Age & $0.000(-0.006$ to 0.007$)$ & 0.95 & - & - \\
\hline Fever duration & $0.8(0.23$ to 1.37$)$ & 0.006 & - & - \\
\hline Serum white blood cells & 0.29 (0.15 to 0.42$)$ & $<0.001$ & 1.13 (1.01 to 1.26$)$ & 0.040 \\
\hline Log plasma neutrophil gelatinase-associated lipocalin & $0.29(0.21$ to 0.36$)$ & $<0.001$ & $3.08(1.36$ to 6.96$)$ & 0.007 \\
\hline Log serum C-reactive protein & $0.20(0.14$ to 0.27$)$ & $<0.001$ & $2.11(0.91$ to 4.9$)$ & 0.084 \\
\hline Hydronephrosis & $0.05(-0.13$ to 0.23$)$ & 0.593 & - & - \\
\hline Vesicoureteral reflux & $0.23(-0.01$ to 0.46$)$ & 0.062 & - & - \\
\hline
\end{tabular}

$\mathrm{Cl}$, confidence interval; OR, odds ratio.

antibiotic therapy. Yilmaz et al. (1) also demonstrated that uNGAL levels were higher in the UTI group than the control group. However, Piccoli et al. (30) reported conflicting data for using uNGAL levels to detect APN in 50 adult patients. While no statistical difference in UNGAL levels was found between patients with and without APN, the median UNGAL tended to be higher in patients with magnetic resonance-proven APN.

NGAL is also emerging as an excellent urine and plasma biomarker for early prediction, monitoring, and determining the prognosis of AKI in several common clinical scenarios (5-7). It is transiently expressed in developing nephrons and induces the mesenchymal-epithelial transition, leading to the conversion of metanephric tissue into the glomeruli and proximal renal tubules (31). Therefore, NGAL appears to play an important role in the repair and regeneration of kidney tubule cells after AKI. Both AKI and APN are characterized by acute, mostly tubulointerstitial kidney damage, although the damage is diffuse in AKI and limited to a section of the renal 


\section{Articles | Simet al.}

a

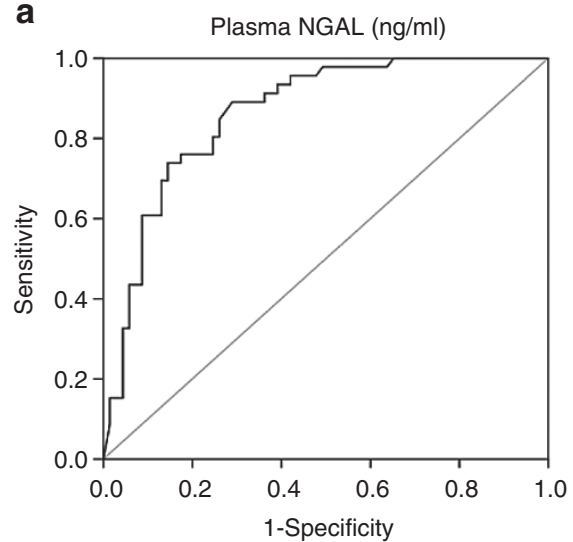

d

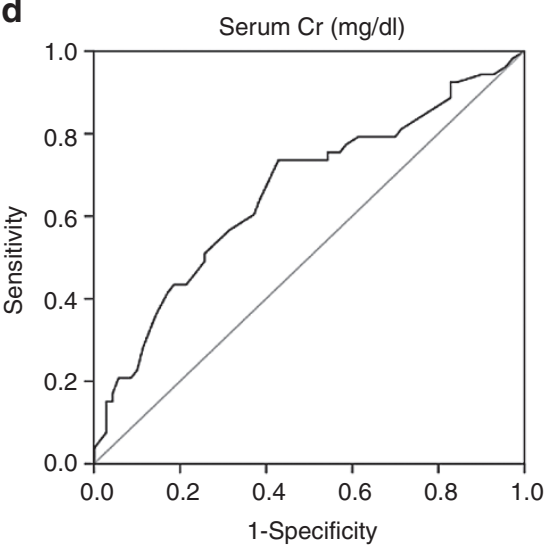

b

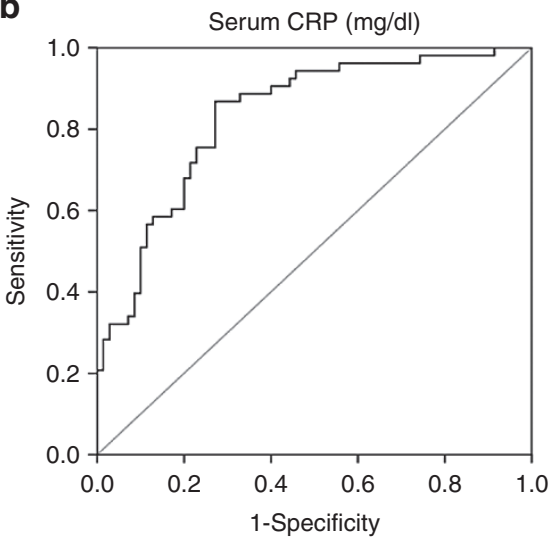

e

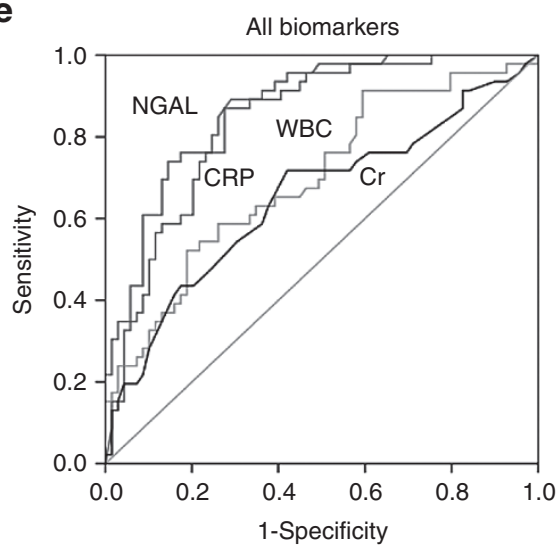

c

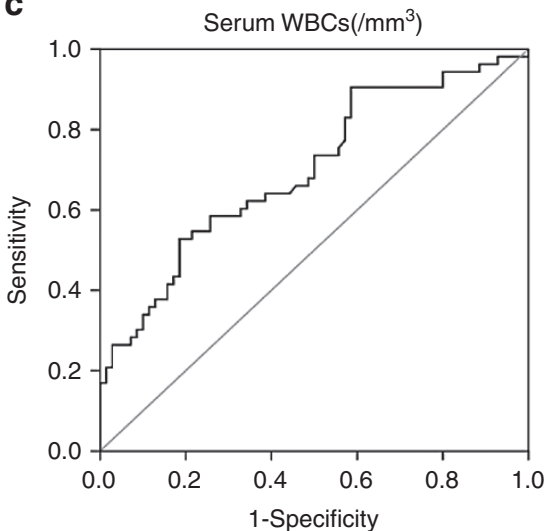

Figure 4. Receiver operating curve for the prediction of acute pyelonephritis. (a) For plasma neutrophil gelatinase-associated lipocalin (area under the curve $(A U C)=0.864)$. (b) For serum C-reactive protein (AUC $=0.842)$. (c) For serum white blood cells $(A U C=0.698)$. (d) For serum Cr (AUC $=0.650)$. (e) All biomarkers (all $P<0.05$ ).

parenchyma in APN (30). In adult patients with established AKI, uNGAL levels have been reported to effectively differentiate between prerenal and intrinsic AKI (7). Likewise, we found in a previous study that urine and plasma NGAL levels could differentiate APN from a lower UTI in children with febrile UTIs (AUC-ROC 0.78 and 0.748 , respectively) $(18,19)$. The present study lends support to our previous findings, showing a higher AUC value for NGAL for differentiating between APN and lower UTI (AUC-ROC, 0.864). While both our previous and present studies raised the possibility that plasma NGAL levels reflected renal involvement even without evidence of AKI, the optimal cut-off values of plasma NGAL for diagnosing APN were somewhat different from our previous study. In the present study, the best cut-off value for predicting APN was $102.5 \mathrm{ng} / \mathrm{ml}$ with a sensitivity of $89 \%$ and a specificity of $71 \%$, whereas in the previous study, the ideal cut-off value was $61.0 \mathrm{ng} / \mathrm{ml}$ with a sensitivity of $75 \%$ and a specificity of $78 \%$ (19). Different sampling collection techniques might have caused these dissimilar results. Previously, the plasma NGAL levels were measured bedside, directly after addition of several drops of whole blood to a disposable device without using ethylenediaminetetraacetic acid (EDTA)-anticoagulated tubes (19). In the present study, levels were measured in EDTAanticoagulated whole blood specimens using the Triage NGAL assay. A significant source of variation in NGAL levels likely arose in the period from sampling to measurement (between measuring in EDTA plasma and measuring by directly adding several drops of whole blood to the device). Similarly, a clinically important and statistically significant bias has been demonstrated between NGAL measured in EDTA plasma and serum in patients admitted to intensive care (32). The suggested cut-off value of NGAL for APN in our present study could not be compared with the findings of other groups because data were unavailable. In critically ill children with septic shock, an optimal cut-off value for the prediction of AKI was $139 \mathrm{ng} / \mathrm{ml}$ (sensitivity $86 \%$, specificity $39 \%$, positive predictive value $39 \%$, negative predictive value $94 \%$ ) (33). For the 2 -h plasma NGAL measurement after pediatric cardiopulmonary bypass surgery, the AUC was 0.96 , sensitivity was $84 \%$, and specificity was $94 \%$ for prediction of AKI using a cut-off value of $150 \mathrm{ng} / \mathrm{ml}$ (34). Critically ill adult patients also showed a similar cut-off value of plasma NGAL for the prediction of AKI $(35,36)$. Therefore, these findings suggest that, in some cases, the optimal cut-off value of plasma NGAL for predicting APN can be comparable to that determined for AKI.

In the present study, gender, fever duration before antibiotic treatment, serum WBCs, serum Cr, log serum CRP, and log plasma NGAL were significantly associated with the presence of APN in the univariate analysis; however, in a multivariate stepwise logistic regression analysis, only log plasma NGAL 
and serum leukocyte counts were remained as independent predictors of APN. Log plasma NGAL level showed the lowest $P$ value among variables, and log serum CRP no longer remained a significant predictor for APN. We also found a positive correlation between levels of plasma NGAL and other laboratory and clinical parameters in patients with symptomatic UTIs. Log plasma NGAL levels were strongly correlated with log serum CRP, serum levels of leukocyte counts and Cr, as well as fever duration before admission. According to ROC analyses, serum levels of CRP, WBCs, and $\mathrm{Cr}$ were predictive for the presence of APN in patients with UTIs. The CRP AUC was not significantly different from that of plasma NGAL, but the AUCs of WBC and Cr were lower compared to the NGAL AUC. Therefore, increased NGAL, CRP, WBCs, and Cr in blood may suggest the presence of APN in children with UTIs. Among these variables, increased plasma NGAL could be the most important sensitive and direct marker of APN, indicating renal damage even in the presence of normal glomerular filtration. Notably, uNGAL/Cr was also significantly correlated with other biomarkers, such as kidney injury molecule-1 and cystatin C, as well as serum levels of CRP and WBC count in children with febrile UTIs $(n=73)(18)$. These results are different from preliminary findings in our previous study. Relatively small numbers of enrolled patients $(n=47)$ in the previous study might be a plausible explanation for the difference between studies (19).

In this study, there were no differences in plasma NGAL levels between patients with and without scars or between those with and without VUR. This was consistent with our previous findings $(18,19)$. Conflicting data have shown that uNGAL is elevated in patients with renal scarring compared to the level in those without scarring $(1,37)$. In patients diagnosed with VUR without evidence of current UTI, uNGAL levels were also significantly higher compared to those in controls (37). The acute infectious condition in our present study may have affected the results, and the small number of patients with VUR or renal scarring could be a limitation when trying to interpret and compare these different findings to other populations.

Taken together, early measurement of plasma NGAL levels could be very useful for identifying patients with renal involvement in symptomatic UTIs. The NGAL levels may also be a useful tool for predicting treatment response of children with UTIs. We may integrate these findings into the current management algorithms of pediatric UTIs. For example, the routine use of imaging for the localization of UTI could be excluded in children with low plasma NGAL levels. Increased plasma NGAL levels can be helpful in differentiating APN from a lower UTI in patients with UTIs. In patients with suspected UTI high NGAL levels would be also greatly helpful for early initiation of treatment and evaluation. Although the diagnosis of UTI is made on the basis of pyuria and quantitative urine culture results (20), negative urine cultures are not uncommon in patients with UTIs. Levtchenko et al. (38) reported that $9 \%$ of 166 children had negative urine culture findings despite clini$\mathrm{cal}$ and scintigraphic evidence of APN. Alarmingly, in different studies of adult patients hospitalized with a diagnosis of APN, urinary cultures were positive only in 23.5 and $30.7 \%$ of the patients, respectively $(21,39)$. Furthermore, the selection of oral or intravenous antibiotics may be directed by plasma NGAL levels as well as the clinical course of the disease. In the longterm, performing voiding cystourethrogram could be reconsidered in cases with low plasma NGAL levels, as they suggest the presence of lower UTIs. Persistently high NGAL levels may be also related to insufficient treatment of symptomatic UTIs in children even without AKI. A follow-up DMSA scan could be highly recommended to exclude renal scarring in cases when the plasma NGAL elevation lasts for longer periods.

There were some limitations to this study. First, we did not have a baseline plasma NGAL level in healthy children. Second, this was a single-center study of pediatric patients with symptomatic UTIs during hospitalization. Although we enrolled children aged from 1 mo to $5 \mathrm{y}$, the median age of patients was less than 12 mo. Our results will need to be validated in a larger, multicenter, prospective study of various age groups. Third, a relatively small study population and the presence of acute infection may have been obstacles for the precise evaluation of plasma NGAL for predicting VUR and renal scarring.

Early identification of renal parenchymal involvement and timely initiation of treatment are invaluable for preventing renal injury in children with UTIs. In this study, we investigated the association between plasma NGAL, one of the most promising biomarkers for AKI, and APN in children with symptomatic UTIs. Our findings showed that plasma NGAL does have great promise for therapeutic monitoring and diagnostic value for the acute phase of APN in children even without AKI, but that plasma levels may not play significant roles for the prediction of renal scarring or the presence of VUR. Further investigations in a larger population are required to confirm the potential application of plasma NGAL levels for diagnosing and managing UTIs in children.

\section{METHODS}

\section{Patients}

This study was conducted at our Department of Pediatrics from October 2013 to September 2014. Children aged from 1 mo to $5 \mathrm{y}$ with their first symptomatic UTI were considered for enrollment. Symptoms included a body temperature $\geq 38^{\circ} \mathrm{C}$, loin pain, gross hematuria, dysuria, or voiding frequency. If the following findings of UTI were present, UTI was diagnosed: pyuria $(\geq 5 \mathrm{WBCs} /$ high-power field) and positive urine cultures (pure growth of $>10^{5}$ organisms/ $\mathrm{ml})$. Patients with UTIs were divided into an APN group and lower UTI group according to the presence of renal cortical defects on the DMSA scan. Patients with urinary tract anomalies, except VUR, or a history of previous UTIs were excluded. Urine samples for culture were obtained by catheterization or suprapubic aspiration for nontoilet-trained children and from a clean voided midstream for the other patients. This study was approved by our institutional review board before initiation. Written informed consent was obtained from the parents of each child before enrollment.

\section{Laboratory and Radiological Assessments}

Blood levels of leukocytes, CRP, Cr, and NGAL were measured at admittance and after 4-5 d of intravenous antibiotic treatment. Plasma NGAL was measured in EDTA-anticoagulated whole blood specimens using the Triage NGAL assay (Alere, San Diego, CA). The assay had a detection range of $15-1,300 \mathrm{ng} / \mathrm{ml}$. Renal sonography and DMSA scintigraphy were performed upon UTI diagnosis. Hydronephrosis 
was diagnosed according to the Society for Fetal Urology's classification (40), and APN was defined as the presence of focal or diffuse areas of decreased cortical uptake shown by the DMSA scan. If the first scintigraphic result was abnormal, then a repeat examination was performed 5-6 mo after the initial study to assess the presence of persistent renal lesions or scars. A voiding cystourethrogram was performed before discharge. Laboratory, clinical, and imaging results were compared between the APN and lower UTI groups.

\section{Statistical Analyses}

Statistical comparisons were performed with the SPSS program for Windows (version 18.0, SPSS, Chicago, IL). Categorical data are presented as numbers (\%) and continuous data as the mean \pm SD or medians with corresponding 25 th and 75 th percentiles (IQR). Normally distributed continuous variables were compared with Student's $t$-test, whereas non-normally distributed continuous variables were compared with the Mann-Whitney $U$-test. The $\chi^{2}$ test was performed for the evaluation of qualitative data. To adjust for age and gender, the ANCOVA test was used. Before correlations were tested, non-normally distributed values (NGAL and CRP) were log-transformed to better approximate normal distributions. All factors that might predict APN were tested with univariate analysis. Then, variables which had a $P$ value of $\leq 0.05$ were included in the multivariate analysis. In this stage, backward stepwise logistic regression analysis was carried out and variables which had a $P$ value of $\geq 0.1$ were removed from the model. Correlations between log plasma NGAL and other variables were evaluated by Pearson's partial correlation test ( $r=$ partial correlation coefficient adjusted by age and gender). ROC analysis was performed, and the AUC was calculated to identify the best cut-off values for plasma NGAL, CRP, leukocyte counts, and Cr to detect APN. The differences between each variable's ability to detect APN, measured by AUCs, were evaluated with Medcalc (version 13.1.1, MedCalc Software, Acacialaan, Ostend, Belgium). Differences were considered significant if $P<0.05$.

\section{STATEMENT OF FINANCIAL SUPPORT}

No financial assistance was received to support this study.

\section{REFERENCES}

1. Yilmaz A, Sevketoglu E, Gedikbasi A, et al. Early prediction of urinary tract infection with urinary neutrophil gelatinase associated lipocalin. Pediatr Nephrol 2009;24:2387-92.

2. Montini G, Tullus K, Hewitt I. Febrile urinary tract infections in children. N Engl J Med 2011;365:239-50.

3. Rushton HG, Majd M. Dimercaptosuccinic acid renal scintigraphy for the evaluation of pyelonephritis and scarring: a review of experimental and clinical studies. J Urol 1992;148(5 Pt 2):1726-32.

4. Rushton HG. The evaluation of acute pyelonephritis and renal scarring with technetium 99m-dimercaptosuccinic acid renal scintigraphy: evolving concepts and future directions. Pediatr Nephrol 1997;11:108-20.

5. Bolignano D, Donato V, Coppolino G, et al. Neutrophil gelatinaseassociated lipocalin (NGAL) as a marker of kidney damage. Am J Kidney Dis 2008;52:595-605.

6. Wheeler DS, Devarajan P, Ma Q, et al. Serum neutrophil gelatinase-associated lipocalin (NGAL) as a marker of acute kidney injury in critically ill children with septic shock. Crit Care Med 2008;36:1297-303.

7. Seibert FS, Pagonas N, Arndt R, et al. Calprotectin and neutrophil gelatinaseassociated lipocalin in the differentiation of pre-renal and intrinsic acute kidney injury. Acta Physiol (Oxf) 2013;207:700-8.

8. Bolignano D, Coppolino G, Campo S, et al. Neutrophil gelatinaseassociated lipocalin in patients with autosomal-dominant polycystic kidney disease. Am J Nephrol 2007;27:373-8.

9. Brunner HI, Mueller M, Rutherford C, et al. Urinary neutrophil gelatinaseassociated lipocalin as a biomarker of nephritis in childhood-onset systemic lupus erythematosus. Arthritis Rheum 2006;54:2577-84.

10. Rabb H. Novel urinary markers for early diagnosis of ARF. Am J Kidney Dis 2003;42:599-600.

11. Mishra J, Mori K, Ma Q, et al. Amelioration of ischemic acute renal injury by neutrophil gelatinase-associated lipocalin. J Am Soc Nephrol 2004;15:3073-82.
12. Flo TH, Smith KD, Sato S, et al. Lipocalin 2 mediates an innate immune response to bacterial infection by sequestrating iron. Nature 2004;432: 917-21.

13. Goetz DH, Holmes MA, Borregaard N, Bluhm ME, Raymond KN, Strong RK. The neutrophil lipocalin NGAL is a bacteriostatic agent that interferes with siderophore-mediated iron acquisition. Mol Cell 2002;10:1033-43.

14. Nguyen MT, Devarajan P. Biomarkers for the early detection of acute kidney injury. Pediatr Nephrol 2008;23:2151-7.

15. Björkqvist M, Källman J, Fjaertoft G, Xu S, Venge P, Schollin J. Human neutrophil lipocalin: normal levels and use as a marker for invasive infection in the newborn. Acta Paediatr 2004;93:534-9.

16. Fjaertoft G, Foucard T, Xu S, Venge P. Human neutrophil lipocalin (HNL) as a diagnostic tool in children with acute infections: a study of the kinetics. Acta Paediatr 2005;94:661-6.

17. Ichino $M$, Kuroyanagi $Y$, Kusaka $M$, et al. Increased urinary neutrophil gelatinase associated lipocalin levels in a rat model of upper urinary tract infection. J Urol 2009;181:2326-31.

18. Yim HE, Yim H, Bae ES, Woo SU, Yoo KH. Predictive value of urinary and serum biomarkers in young children with febrile urinary tract infections. Pediatr Nephrol 2014;29:2181-9.

19. Seo WH, Nam SW, Lee EH, Je BK, Yim HE, Choi BM. A rapid plasma neutrophil gelatinase-associated lipocalin assay for diagnosis of acute pyelonephritis in infants with acute febrile urinary tract infections: a preliminary study. Eur J Pediatr 2014;173:229-32.

20. Subcommittee on Urinary Tract Infection, Steering Committee on Quality Improvement and Management. Roberts KB. Urinary tract infection: clinical practice guideline for the diagnosis and management of the initial UTI in febrile infants and children 2 to 24 months. Pediatrics 2011;128: 595-610.

21. Piccoli GB, Consiglio V, Deagostini MC, et al. The clinical and imaging presentation of acute "non complicated" pyelonephritis: a new profile for an ancient disease. BMC Nephrol 2011;12:68.

22. Talner LB, Davidson AJ, Lebowitz RL, Dalla Palma L, Goldman SM. Acute pyelonephritis: can we agree on terminology? Radiology 1994;192:297-305.

23. Piccoli GB, Consiglio V, Colla L, et al. Antibiotic treatment for acute 'uncomplicated' or 'primary' pyelonephritis: a systematic, 'semantic revision'. Int J Antimicrob Agents 2006;28:Suppl 1:S49-63.

24. Leroy S, Fernandez-Lopez A, Nikfar R, et al. Association of procalcitonin with acute pyelonephritis and renal scars in pediatric UTI. Pediatrics 2013;131:870-9.

25. Kjeldsen L, Johnsen AH, Sengeløv H, Borregaard N. Isolation and primary structure of NGAL, a novel protein associated with human neutrophil gelatinase. J Biol Chem 1993;268:10425-32.

26. Sevéus L, Amin K, Peterson CG, Roomans GM, Venge P. Human neutrophil lipocalin (HNL) is a specific granule constituent of the neutrophil granulocyte. Studies in bronchial and lung parenchymal tissue and peripheral blood cells. Histochem Cell Biol 1997;107:423-32.

27. Supavekin S, Zhang W, Kucherlapati R, Kaskel FJ, Moore LC, Devarajan P. Differential gene expression following early renal ischemia/reperfusion. Kidney Int 2003;63:1714-24.

28. Xu S, Venge P. Lipocalins as biochemical markers of disease. Biochim Biophys Acta 2000;1482:298-307.

29. Hatipoglu S, Sevketoglu E, Gedikbasi A, et al. Urinary MMP-9/NGAL complex in children with acute cystitis. Pediatr Nephrol 2011;26:1263-8.

30. Piccoli GB, Ferraresi M, Aroasio E, Gonella S, De Pascale A, Veltri A. The search for perfect biomarkers in acute kidney damage: the case of NGAL, from AKI to acute pyelonephritis: back to the clinic? Nephrol Dial Transplant 2012;27:3665-6.

31. Mori K, Nakao K. Neutrophil gelatinase-associated lipocalin as the realtime indicator of active kidney damage. Kidney Int 2007;71:967-70.

32. Itenov TS, Bangert K, Christensen PH, Jensen JU, Bestle MH; Procalcitonin and Survival Study (PASS)-study group. Serum and plasma neutrophil gelatinase associated lipocalin (NGAL) levels are not equivalent in patients admitted to intensive care. J Clin Lab Anal 2014;28:163-7.

33. Wheeler DS, Devarajan P, Ma Q, et al. Serum neutrophil gelatinaseassociated lipocalin (NGAL) as a marker of acute kidney injury in critically ill children with septic shock. Crit Care Med 2008;36:1297-303. 
34. Dent CL, Ma Q, Dastrala S, et al. Plasma neutrophil gelatinase-associated lipocalin predicts acute kidney injury, morbidity and mortality after pediatric cardiac surgery: a prospective uncontrolled cohort study. Crit Care 2007;11:R127.

35. Constantin JM, Futier E, Perbet S, et al. Plasma neutrophil gelatinase-associated lipocalin is an early marker of acute kidney injury in adult critically ill patients: a prospective study. J Crit Care 2010;25:176.e1-6.

36. Cruz DN, de Cal M, Garzotto F, et al. Plasma neutrophil gelatinaseassociated lipocalin is an early biomarker for acute kidney injury in an adult ICU population. Intensive Care Med 2010;36:444-51.

37. Ichino M, Kusaka M, Kuroyanagi $Y$, et al. Urinary neutrophilgelatinase associated lipocalin is a potential noninvasive marker for renal scarring in patients with vesicoureteral reflux. J Urol 2010;183: 2001-7.

38. Levtchenko EN, Lahy C, Lévy J, Ham HR, Piepsz A. Role of Tc-99m DMSA scintigraphy in the diagnosis of culture negative pyelonephritis. Pediatr Nephrol 2001;16:503-6.

39. Rollino C, Beltrame G, Ferro M, Quattrocchio G, Sandrone M, Quarello F. Acute pyelonephritis in adults: a case series of 223 patients. Nephrol Dial Transplant 2012;27:3488-93.

40. Fernbach SK, Maizels M, Conway JJ. Ultrasound grading of hydronephrosis: introduction to the system used by the Society for Fetal Urology. Pediatr Radiol 1993;23:478-80. 